\title{
More Metalwork Removals of Olecranon Fractures After Tension Banding Wiring than Plate Fixation-A Propensity Score Matching Analysis
}

\section{Yi Li}

Huizhou municipal central hospital

Longhai Qiu

Huizhou municipal central hospital

\section{Hongbo Wu}

Huizhou municipal central hospital

\section{Ruixiong Chen}

Huizhou municipal central hospital

\section{Zhiwen Zhang}

Huizhou municipal central hospital

\section{Xiaofeng Wang}

Huizhou municipal central hospital

\section{Yuliang Huang ( $\sim$ huangyuliang2020@126.com )}

City Hospital: Hospital Municipal

\section{Research article}

Keywords: olecranon fracture, elbow instability, complications, Mayo elbow performance score, metalwork removal

Posted Date: January 27th, 2021

DOI: https://doi.org/10.21203/rs.3.rs-154380/v1

License: (c) (i) This work is licensed under a Creative Commons Attribution 4.0 International License. Read Full License

Version of Record: A version of this preprint was published at BMC Musculoskeletal Disorders on August 13th, 2021. See the published version at https://doi.org/10.1186/s12891-021-04559-0. 


\section{Abstract}

Background: Traditional tension band wiring and plate fixation represent the most common methods for treating olecranon fractures. However, there is no agreement as to which method provides the best outcome. The aim of this retrospective study was to compare the outcomes of tension band wiring (TBW) and plate fixation (PF) for treating displaced olecranon fractures. This is the first study to use propensity score matching analysis to compare treatment methods for olecranon fracture.

Method: A total of 107 patients aged between 18 and 85 years old had acute isolated and displaced olecranon fractures. The patients were divided into either TBW $(n=49)$ or PF $(n=58)$ groups. To conduct propensity score matching for the treatment method (TBW versus PF), 58 patients were analyzed by logistic regression (29 patients in each group). Various demographic and treatment-related variables were examined and analyzed to determine their correlation.

Results: Functional effects between two groups are similar( in terms of Mayo Elbow Performance Score (MEPS), the patients' range of elbow motion (ROM) and forearm rotation (RFR), the time return to work (RTW)). The total adverse events rate and metalwork removal events rate are higher in TBW than that in PF. After propensity score matching analysis, the similar primary treatment efficacy (indicated by excellent MEPS) and higher primary adverse events rate ( indicated by metalwork removal) were perceived in TBW than that in PF. Logistic regression analysis revealed that fracture type was an independent factor that affected the efficacy of a treatment (regression coefficient $=-1.24<0$, $\mathrm{P}=0.03$ ), indicating that fracture severity was inversely proportional to the efficacy of a treatment for olecranon fracture. Furthermore, logistic regression analysis demonstrated that the treatment method was an independent factor that affected metalwork removal of olecranon fracture (regression coefficient $2.38>0, O R=10.77, P<0.01$ ), indicating that the risk of metalwork removal in the TBW Group was 10.77 times that in the PF Group.

Conclusion: The results indicated that plate fixation should be the first choice of treatment method for olecranon fractures, especially in younger patients with higher quality of life requirements.

Trial regislation: The study was not registered in the clinical trial registration center, because it was a retrospective case-control study, which was an observational study with no intervention measures.

Level of evidence: Level III; Retrospective Cohort Comparison; Treatment Study

\section{Background}

The proximal ends of the radius and ulna bones come together with the distal end of the humerus at the elbow. The proximal ulna bone is called the olecranon, and it forms the bony tip of the elbow when the elbow bends. A hinge joint is shaped by the top side of the olecranon and the distal end of the humerus[1]. The olecranon is situated directly under the skin and is vulnerable to damage. Olecranon fractures account for $1 \%$ of all upper extremity fractures, with an incidence rate of 11.5 to 12 per 100,000 population annually[1, 2].

Most olecranon fractures involve the articular surface of the elbow joint, and uneven articular surfaces can cause limited elbow joints, traumatic arthritis and other complications[3, 4]. Nonsurgical treatment, usually involving plaster immobilization, is recommended for patients with nondisplaced fractures or minimally displaced fractures, where the gap or step-off between fragments is less than $2 \mathrm{~mm}$ and the extensor mechanism is intact. Otherwise, surgical intervention is necessary to achieve accurate reduction and rigid fixation in cases of unstable elbow joints and osteoarthritis[1,5]. There are many methods of surgical intervention for the treatment of olecranon fractures, including standard tension band wiring, modified tension band wiring, anatomical plate, $1 / 3$ tube type plate, hollow nail plus 
tension and memory alloy[1, 6, 7]. However, standard tension band wiring (TBW) still remains the gold standard in the treatment of olecranon fractures[3], especially in Mayo type 2A fractures. The aim of this method is to convert the extensor forces of the triceps muscle into compressive forces along the articular surface to compress the fracture. Some researchers believed that plate fixation (PF) is an effective alternative, as there are few complications with this method. PF can be used for any type of olecranon fracture but is particularly recommended for the following indications: comminuted fractures, Monteggia fracture dislocations, oblique fractures (particularly those distal to midpoint of the trochlear notch) and fractures that involve the coronoid process[5]. However, it is unclear whether TBW and PF provides the best outcome for simply displaced olecranon fractures.

Therefore, the purpose of the current study was to examine the differences between TBW and PF using propensity score matching analysis and to propose appropriate treatment methods for simply displaced olecranon fractures. To our knowledge, this is the first study to adopt propensity score matching to compare the functional effects of and adverse events related to the use of TBW vs PF for the treatment of simply displaced fractures of the olecranon. With propensity score matching, differences in baseline factors between groups are eliminated.

\section{Methods}

This was a retrospective single-center case control trial including active adult patients with a simply displaced fracture of the olecranon treated at Huizhou Central People's Hospital (Huizhou Hospital Affiliated to Guangdong Medical University). Ethical approval was obtained from the Institutional Review Board of Guangdong Medical University, and the study conformed to the tenets of the Declaration of Helsinki.

\section{Patients}

In total, 176 patients with displaced olecranon fractures (Mayo 2A and Mayo2B) were treated at our department between 2005 and 2019. 69 patients who did not follow up for more than 3 months after treatment were excluded from

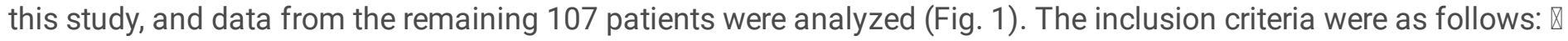

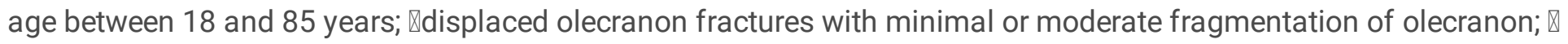
olecranon fractures treated by TBW or PF. The exclusion criteria were as follows: $\otimes$ pregnant women/skeletal immature patients; $\otimes$ patients who were unable to comply with follow-up; $₫$ associated fractures with radial head fracture/coronoid/distal aspects of the humerus; $\nabla$ associated ligamentous injury/dislocation/subluxation of the elbow; $₫$ previous history of fractures on the side of injury; $\Downarrow$ open olecranon fractures.

\section{Variables}

The following clinical factors were examined retrospectively using the patients' medical records: age; sex; career (heavy manual worker or not); mechanism of injury (fall from standing height; fall from height; motor vehicle collision; sports; fight/assault); dominant hand; side of injury; body mass index (BMI); diabetes; alcohol consumption (alcohol consumption < 21 units/wk or alcohol consumption > 21 units/wk); smoking; American Society of Anesthesiologists(ASA) grade; associated injury (ipsilateral shoulder joint injury, ipsilateral wrist joint injury, minor head, others); type of fracture (classified by Mayo classification:); intervention method (tension band wiring versus internal plate fixation). The fracture type was divided into 2 categories: Mayo 2A and Mayo 2B. Any disagreements regarding the radiographs and the Mayo classification were resolved by discussion. The patients were divided into 2 categories based on treatment methods: the tension band wiring group (TBW Group: 48 patients) and the plate fixation group (PF Group: 57 patients). The choice of implant was decided by the patients on the advice of the attending surgeons. 


\section{Outcome}

Various functional effects was assessed as following: Mayo Elbow Performance Score (MEPS), the patients' range of elbow motion (ROM) and forearm rotation (RFR), the time return to work (RTW). The MEPS can be divided into 4 levels: excellent $(\geq 90)$, good $(80 \sim 90)$, general $(70 \sim 80)$ and poor $(\geq 70)$. The primary functional outcome was assessed by treatment efficacy ( as indicated by an excellent MEPS, a generic and upper-limb specific validated clinical score). And then the occurrences of various adverse events were recorded as following: metalwork removal, infection, revision and others (union, malunion, painful stiff elbow, persistent instability of the elbow, posttraumatic arthritis, heterotopic ossification). The primary adverse event was assessed by metalwork removal, a frequent complication predominated by prominence at the site of the proximal ulna.

\section{Statistical analysis}

All statistical analyses were performed using SPSS software. First, the baseline data of the two groups were compared. Two-sample independent t-tests were adopted for continuous variables that followed a normal distribution, and twosample independent rank sum tests were adopted otherwise. Chi-square test was used to compare categorical variables. Functional effects between two groups were compared by the Mann-Whitney test because the continuous data were not normally distributed. Adverse events, including various complications, were compared by the Chi-square test. Furthermore, propensity score analysis was performed to minimize selection biases associated with a retrospective data analysis between the TBW Group and the PF Group. For each patient, a propensity score for the treatment method group was calculated by a logistic regression analysis of all predictive variables. Propensity score matching was calculated for all 107 patients by logistic regression analysis for all 13 factors associated with treatment. The concordance index was 0.02 , indicating a strong ability to differentiate patients based on whether they were treated using PF or TBW. The Hosmer-Lemeshow statistic was nonsignificant $(P=0.05)$, indicating good calibration. The propensity scores in the PF Group ranged from 0.153 to 0.788 , and the propensity scores in the TBW Group ranged from 0.153 to 0.788 . The 58 propensity score-matched cases (PF versus TBW) were then evaluated by univariate analysis and multivariate analysis by logistic regression to identify the potential factors associated with 2 primary outcomes:MEPS (functional effects) and metalwork removal (adverse events). In all analyses, two-tailed $p$ values $<0.05$ were considered statistically significant (Fig. 1).

\section{Results}

\section{Comparison of variables and outcomes between Group TBW and Group PF in the original 107 patients}

The variables in the TBW Group and the PF Group in the original 107 patients are shown in Table 1. There were some differences between the TBW Group and the PF Group regarding diabetes, dominant hands and the side of injury. As shown in Table 2, there was no significant difference between the TBW Group and the PF Group in terms of MEPS (91.60 (86.7,94.75) vs $92.2(77.2,95.2), \mathrm{P}=0.56)$, flexion of elbow $(144(131,151)$ vs $145(1335,153.1), \mathrm{P}=0.36)$, forearm rotation $(146(135,150)$ vs 145 (135,150), $\mathrm{P}=0.72)$, and RTW $(6(3,9)$ vs $5(3,7), \mathrm{P}=0.13)$. Interestingly, a significantly different fare was found between the TBW Group and the PF Group: the fare in PF Group was markedly higher than that in the TBW Group $(23204.5(18400,24847)$ vs $11592(9832,17102), P<0.001))$. The total adverse events rate was higher in the TBW Group than in PF Group (75.93\% vs 29.63\%, P<0.001) (Table 3). Moreover, metalwork removal rate was higher in the TBW Group than in the PF Group (48.15\% vs $20.37 \%, \mathrm{P}<0.001)$, and other adverse events rate was higher in the PF Group than in the TBW Group $(12.96 \%$ vs $9.25 \%, \mathrm{P}<0.001)$. 
Table 1

Comparison of variables in Group TB and Group PF in the original 107 patients

\begin{tabular}{|c|c|c|c|c|c|c|c|}
\hline & & Group & & & & & \\
\hline & & TBW & PF & Total & Proportion(\%) & $\begin{array}{l}\text { Chi- } \\
\text { square } \\
\text { value/Z }\end{array}$ & $P$ \\
\hline \multirow[t]{2}{*}{ Sex } & Men & 24 & 34 & 58 & 41.38 & \multirow[t]{2}{*}{0.62} & \multirow[t]{2}{*}{0.43} \\
\hline & Women & 24 & 25 & 49 & 48.98 & & \\
\hline Age & & $\begin{array}{l}35(22 \sim \\
59.8)\end{array}$ & $\begin{array}{l}42(32 \sim \\
56)\end{array}$ & & & -1.24 & 0.22 \\
\hline \multirow[t]{2}{*}{ Occupation } & Heavy worker & 13 & 16 & 29 & 44.83 & \multirow[t]{2}{*}{0} & \multirow[t]{2}{*}{0.99} \\
\hline & No heavy worker & 35 & 43 & 78 & 44.87 & & \\
\hline \multirow{5}{*}{$\begin{array}{l}\text { Associated } \\
\text { injury }\end{array}$} & None & 22 & 33 & 55 & 40.00 & \multirow[t]{5}{*}{5} & \multirow[t]{5}{*}{0.29} \\
\hline & $\begin{array}{l}\text { Ipsilateral shoulder } \\
\text { joint injury }\end{array}$ & 5 & 9 & 14 & 35.71 & & \\
\hline & $\begin{array}{l}\text { Ipsilateral wrist joint } \\
\text { injury }\end{array}$ & 7 & 2 & 9 & 77.78 & & \\
\hline & Minor head injury & 8 & 9 & 17 & 47.06 & & \\
\hline & Others & 6 & 6 & 12 & 50.00 & & \\
\hline \multirow[t]{5}{*}{$\begin{array}{l}\text { Mechanism of } \\
\text { injury }\end{array}$} & $\begin{array}{l}\text { Fall from standing } \\
\text { height }\end{array}$ & 19 & 22 & 41 & 46.34 & \multirow[t]{5}{*}{2.06} & \multirow[t]{5}{*}{0.73} \\
\hline & Fall from height & 6 & 12 & 18 & 33.33 & & \\
\hline & $\begin{array}{l}\text { Motor vehicle } \\
\text { collision }\end{array}$ & 11 & 15 & 26 & 42.31 & & \\
\hline & Sports & 7 & 5 & 12 & 58.33 & & \\
\hline & Fight/assault & 5 & 6 & 11 & 45.45 & & \\
\hline \multirow[t]{2}{*}{ Diabetes } & No diabetes & 39 & 56 & 95 & 88.79 & \multirow[t]{2}{*}{4.96} & \multirow[t]{2}{*}{0.03} \\
\hline & Diabetes & 9 & 3 & 12 & 11.21 & & \\
\hline \multirow[t]{2}{*}{ Dominant hand } & Left hand & 0 & 6 & 6 & 0.00 & \multirow[t]{2}{*}{5.17} & \multirow[t]{2}{*}{0.03} \\
\hline & Right hand & 48 & 53 & 101 & 47.52 & & \\
\hline \multirow[t]{2}{*}{ Side of injury } & Left hand & 24 & 41 & 65 & 36.92 & \multirow[t]{2}{*}{4.22} & \multirow[t]{2}{*}{0.048} \\
\hline & Right hand & 24 & 18 & 42 & 57.14 & & \\
\hline \multirow[t]{3}{*}{ ASA grade } & 1 & 34 & 41 & 75 & 45.33 & \multirow[t]{3}{*}{-0.14} & \multirow[t]{3}{*}{0.89} \\
\hline & 2 & 10 & 13 & 23 & 43.48 & & \\
\hline & 3 & 4 & 5 & 9 & 44.40 & & \\
\hline \multirow{2}{*}{$\begin{array}{l}\text { Alcohol } \\
\text { consumption }\end{array}$} & $<21$ units/wk & 42 & 55 & 97 & 43.30 & \multirow[t]{2}{*}{1.02} & \multirow[t]{2}{*}{0.34} \\
\hline & $>21$ units/wk & 6 & 4 & 10 & 60.00 & & \\
\hline
\end{tabular}




\begin{tabular}{|c|c|c|c|c|c|c|c|}
\hline & & Group & & & & & \\
\hline \multirow[t]{2}{*}{ Smoking } & No smoking & 34 & 41 & 75 & 45.33 & \multirow[t]{2}{*}{0.023} & \multirow[t]{2}{*}{1.00} \\
\hline & Smoking & 14 & 18 & 32 & 43.75 & & \\
\hline \multirow[t]{2}{*}{ Fracture } & May 2A & 29 & 28 & 57 & 50.88 & \multirow[t]{2}{*}{1.79} & \multirow[t]{2}{*}{0.24} \\
\hline & May 2B & 19 & 31 & 50 & 38.00 & & \\
\hline \multicolumn{2}{|l|}{ BMI } & $\begin{array}{l}23.32 \pm \\
3.29\end{array}$ & $\begin{array}{l}22.47 \pm \\
3.31\end{array}$ & & & 0.04 & 0.19 \\
\hline \multicolumn{2}{|c|}{ Follow period(month) } & $\begin{array}{l}33(14 \sim \\
65)\end{array}$ & $\begin{array}{l}41(16 \sim \\
55)\end{array}$ & & & -0.15 & 0.89 \\
\hline
\end{tabular}

Table 2

Comparison of functional effects between Group TB and Group PF in the original 107 patients

\begin{tabular}{|c|c|c|c|c|}
\hline & Group & M (25\%QSD`75\%QSD) & $\mathbf{Z}$ & $\mathbf{P}$ \\
\hline \multirow[t]{2}{*}{ MEPS } & Tension band wiring & $91.6(86.7,94.75)$ & -0.59 & 0.56 \\
\hline & Plate fixation & $92.2(77.2,95.2)$ & & \\
\hline \multirow[t]{2}{*}{ Elbow flexion } & Tension band wiring & $144(131,151)$ & -0.93 & 0.36 \\
\hline & Plate fixation & $145(133.5,153.1)$ & & \\
\hline \multirow[t]{2}{*}{ Forearm rotation } & Tension band wiring & $146(135,150)$ & -0.37 & 0.72 \\
\hline & Plate fixation & $145(135,150)$ & & \\
\hline \multirow[t]{2}{*}{ RTW(month) } & Tension band wiring & $6(3,9)$ & -1.53 & 0.13 \\
\hline & Plate fixation & $5(3,7)$ & & \\
\hline \multirow[t]{2}{*}{ Fare(yuan) } & Tension band wiring & 11592(9832,17102) & -5.2 & $<0.001$ \\
\hline & Plate fixation & $23204.5(18400,24847)$ & & \\
\hline
\end{tabular}

Table 3

Comparison of adverse events between Group TB and Group PF in the original 107 patients

\begin{tabular}{|llllllll|}
\hline & & TBW & PF & Total & Proportion(\%) & Chi-square value/Z & P \\
\hline Adverse events & Total & 35 & 21 & 51 & 25.49 & 11.5 & $<0.001$ \\
\cline { 2 - 8 } & Infection & 2 & 2 & 4 & 50.00 & 0.44 & 1 \\
\cline { 2 - 8 } & Metalwork removal & 26 & 11 & 37 & 70.27 & 11.8 & $<0.001$ \\
\cline { 2 - 8 } & Revision & 2 & 1 & 3 & 66.67 & 0,593 & 0.59 \\
\cline { 2 - 7 } & Others & 5 & 7 & 12 & 41.67 & 18.44 & $<0.001$ \\
\hline
\end{tabular}

Comparison of functional effects and adverse events between Group TBW and Group PF in $\mathbf{5 8}$ patients after propensity score matching. 
Propensity score matching resulted in 58 patients being matched to 29 patients in each group (Table 4). As indicated by Table 5, there was no significant difference between two groups in terms of fuctional effects, including MEPS (( 93.8 $(87.9,95.5)$ vs 92 (86.8,94.3), $\mathrm{P}=0.93)$, elbow flexion $(148(135,153)$ vs $144(134,151), \mathrm{P}=0.62)$, forearm rotation $(145$ $(135,148.5)$ vs $148(135,150), P=0.5)$ and $\operatorname{RTW}(6(3.5,9)$ vs $6(3,9), P=0.9)$ (Table 5). Interestingly, the fare was higher in Group PF than in Group TBW(23519 $(17232,25424.5)$ vs $11943(10201,17978), P<0.001)$. However, as indicated by Table 6, total adverse events rates was higher in Group TBW than that in Group PF $(79.31 \%$ vs $27.59 \%, P=0.001)$. What's more, metalwork removal was especially higher in Group TBW than that in Group PF (65.56\% vs $17.24 \%, \mathrm{P}=$ 0.03). 
Table 4

Comparison of variables in Group TB and Group PF after propensity match scoring

\begin{tabular}{|c|c|c|c|c|c|c|c|}
\hline \multicolumn{8}{|c|}{ Group } \\
\hline & & TBW & PF & Sum & Odds(\%) & $x^{2} / z$ & $P$ \\
\hline \multirow[t]{2}{*}{ Sex } & Men (\%) & 14 & 18 & 32 & 43.75 & \multirow[t]{2}{*}{1.12} & \multirow[t]{2}{*}{0.21} \\
\hline & Women (\%) & 15 & 11 & 26 & 57.69 & & \\
\hline \multirow[t]{2}{*}{ Occupation } & $\begin{array}{l}\text { No heavy } \\
\text { labor }\end{array}$ & 20 & 20 & 40 & 50.00 & \multirow[t]{2}{*}{0} & \multirow[t]{2}{*}{1} \\
\hline & Heavy labor & 9 & 9 & 18 & 50.00 & & \\
\hline \multirow{5}{*}{$\begin{array}{l}\text { Associated } \\
\text { injury }\end{array}$} & No & 16 & 13 & 29 & 55.17 & \multirow[t]{5}{*}{3.27} & \multirow[t]{5}{*}{$0.55^{\star}$} \\
\hline & $\begin{array}{l}\text { Ipsilateral } \\
\text { shoulder } \\
\text { joint injury }\end{array}$ & 3 & 5 & 8 & 37.50 & & \\
\hline & $\begin{array}{l}\text { Ipsilateral } \\
\text { wrist joints } \\
\text { injury }\end{array}$ & 5 & 2 & 7 & 71.43 & & \\
\hline & $\begin{array}{l}\text { Minor head } \\
\text { injury }\end{array}$ & 3 & 6 & 9 & 33.33 & & \\
\hline & others & 2 & 3 & 5 & 40.00 & & \\
\hline \multirow[t]{5}{*}{$\begin{array}{l}\text { Mechanism } \\
\text { of injury }\end{array}$} & $\begin{array}{l}\text { Fall from } \\
\text { standing } \\
\text { height }\end{array}$ & 14 & 10 & 24 & 58.33 & \multirow[t]{5}{*}{2.08} & \multirow[t]{5}{*}{$0.75^{\star}$} \\
\hline & $\begin{array}{l}\text { Fall from } \\
\text { height }\end{array}$ & 3 & 5 & 8 & 37.50 & & \\
\hline & $\begin{array}{l}\text { Motor vehicle } \\
\text { collision }\end{array}$ & 6 & 9 & 15 & 40.00 & & \\
\hline & Sports & 4 & 3 & 7 & 57.14 & & \\
\hline & Fight/assault & 2 & 2 & 4 & 50.00 & & \\
\hline \multirow[t]{2}{*}{ Diabetes } & No & 27 & 29 & 56 & 48.21 & \multirow[t]{2}{*}{2.07} & \multirow[t]{2}{*}{0.49} \\
\hline & Yes & 2 & 0 & 2 & 100.00 & & \\
\hline $\begin{array}{l}\text { Dominant } \\
\text { hand }\end{array}$ & Right & 29 & 29 & 58 & 50.00 & constant & \\
\hline \multirow[t]{2}{*}{ Side of injury } & Left & 19 & 17 & 36 & 52.78 & \multirow[t]{2}{*}{0.29} & \multirow[t]{2}{*}{0.79} \\
\hline & Right & 10 & 12 & 22 & 45.45 & & \\
\hline \multirow{2}{*}{$\begin{array}{l}\text { Alcohol } \\
\text { consumption }\end{array}$} & $<21$ units/wk & 28 & 26 & 54 & 51.85 & \multirow[t]{2}{*}{1.07} & \multirow[t]{2}{*}{$0.61^{*}$} \\
\hline & $>21$ units/wk & 1 & 3 & 4 & 25.00 & & \\
\hline \multirow[t]{2}{*}{ Smoking } & No & 22 & 20 & 42 & 52.38 & \multirow[t]{2}{*}{0.35} & \multirow[t]{2}{*}{0.78} \\
\hline & Yes & 7 & 9 & 16 & 43.75 & & \\
\hline Fracture & Mayo 2A & 17 & 16 & 33 & 51.52 & 0.07 & 1 \\
\hline
\end{tabular}




\begin{tabular}{|c|c|c|c|c|c|c|c|}
\hline & & Group & & & & & \\
\hline & Mayo 2B & 12 & 13 & 25 & 48.00 & & \\
\hline ASA_grade & & $1(1,1.5)$ & $1(1,1)$ & & & -0.23 & $0.97 \#$ \\
\hline Age & & $33(22,51)$ & $38(30,55.5)$ & & & -1.386 & $0.17 \#$ \\
\hline $\mathrm{BMI}$ & & $22.33(20.85,25.05)$ & $22.9(19.14,25.15)$ & & & -0.156 & $0.88 \#$ \\
\hline Follow period & & $39(15,65)$ & $42(22,53)$ & & & -0.288 & $0.78 \#$ \\
\hline
\end{tabular}

Table 5

Compassion of functional effects between Group TB and Group PF after propensity match scoring

\begin{tabular}{|c|c|c|c|c|}
\hline & & M(25\%QSD,75\%QSD) & $z$ & $\mathbf{P}$ \\
\hline \multirow[t]{2}{*}{ MEPS } & PF & $93.8(87.9,95.5)$ & \multirow[t]{2}{*}{-0.09} & \multirow[t]{2}{*}{0.93} \\
\hline & TB & $92(86.8,94.3)$ & & \\
\hline \multirow[t]{2}{*}{ Elbow of flexion } & PF & $148(135,153)$ & \multirow[t]{2}{*}{-0.51} & \multirow[t]{2}{*}{0.62} \\
\hline & TBW & $144(134,151)$ & & \\
\hline \multirow[t]{2}{*}{ Fore arm rotation } & $\mathrm{PF}$ & $145(135,148.5)$ & \multirow[t]{2}{*}{-0.69} & \multirow[t]{2}{*}{0.50} \\
\hline & TBW & $148(135,150)$ & & \\
\hline \multirow[t]{2}{*}{ RTW (months) } & PF & $6(3.5,9)$ & \multirow[t]{2}{*}{-0.14} & \multirow[t]{2}{*}{0.90} \\
\hline & TBW & $6(3,9)$ & & \\
\hline \multirow[t]{2}{*}{ Fare(yuan) } & PF & $23519(17232,25424.5)$ & \multirow[t]{2}{*}{-3.13} & \multirow[t]{2}{*}{$<0.001$} \\
\hline & TBW & 11943(10201,17978) & & \\
\hline
\end{tabular}

Table 6

Comparison of adverse events between Group TB and Group PF after propensity match scoring

\begin{tabular}{|lllll|}
\hline & PF & TBW & Chi-square value & P \\
\hline Total complications (\%) & $8(27.59)$ & $23(79.31)$ & & 0.001 \\
\hline Infection(\%) & 0 & 1 & $1.018^{*}$ & 1 \\
\hline Metalwork removal (\%) & $5(17.24)$ & $19(65.56)$ & & 0.03 \\
\hline Revision(\%) & $1(34.48)$ & $1(34.48)$ & & 1 \\
\hline Other complication(\%) & $2(6.90)$ & $2(6.90)$ & & 1 \\
\hline
\end{tabular}

Univariate analysis and multivariate analysis of variables relating to treatment efficacy in $\mathbf{5 8}$ patients after propensity match scoring

As indicated by Table 7 and Table 8, the results of univariate analysis of the propensity match scoring revealed that fracture type was significantly correlated with treatment efficacy (as indicated by an excellent MEPS). A multivariate analysis revealed that fracture type was an independent factor that affected the efficacy (regression coefficient=-1.24< 
$0, P=0.03)$, indicating that fracture severity was inversely proportional to the efficacy of the treatment method for an olecranon fracture. The Mayo 2B efficacy rate was $29 \%$ of the Mayo 2A efficacy rate in terms of excellent MEPS. 
Table 7

Univariate analysis of variables relating to treatment efficacy in 58 patients after propensity match scoring

\begin{tabular}{|c|c|c|c|c|c|c|}
\hline & & Excellent & $\begin{array}{l}\text { Not } \\
\text { excellent }\end{array}$ & Odds(\%) & $\chi^{2} / Z / F$ & $\mathbf{P}$ \\
\hline \multirow[t]{2}{*}{ Sex } & Men & 18 & 14 & 54.5 & 0.01 & 1.00 \\
\hline & Women & 15 & 11 & 45.5 & & \\
\hline \multirow[t]{2}{*}{ Occupation } & No heavy labor & 21 & 19 & 63.6 & 1.02 & 0.31 \\
\hline & Heavy labor & 12 & 6 & 36.4 & & \\
\hline \multirow[t]{5}{*}{ Associated injury } & No & 14 & 15 & 42.4 & 2.35 & $0.71^{*}$ \\
\hline & $\begin{array}{l}\text { Ipsilateral shoulder joint } \\
\text { injury }\end{array}$ & 6 & 2 & 18.2 & & \\
\hline & $\begin{array}{l}\text { Ipsilateral wrist joints } \\
\text { injury }\end{array}$ & 4 & 3 & 12.1 & & \\
\hline & Minor head injury & 6 & 3 & 18.2 & & \\
\hline & Others & 3 & 2 & 9.1 & & \\
\hline \multirow{5}{*}{$\begin{array}{l}\text { Mechanism of } \\
\text { injury }\end{array}$} & Fall from standing height & 13 & 11 & 39.4 & 0.95 & $0.95^{\star}$ \\
\hline & Fall from height & 4 & 4 & 12.1 & & \\
\hline & Motor vehicle collision & 9 & 6 & 27.3 & & \\
\hline & Sports & 4 & 3 & 12.1 & & \\
\hline & Fight/Assault & 3 & 3 & 9.1 & & \\
\hline \multirow[t]{2}{*}{ Diabetes } & No & 31 & 25 & 93.9 & 1.57 & $0.501 *$ \\
\hline & Yes & 2 & 0 & 6.1 & & \\
\hline Dominant hand & Right hand & 33 & 25 & 56.9 & constant & \\
\hline \multirow[t]{2}{*}{ Side of injury } & Left hand & 19 & 17 & 57.6 & 0.66 & 0.59 \\
\hline & Right hand & 14 & 8 & 42.4 & & \\
\hline \multirow{2}{*}{$\begin{array}{l}\text { Alcohol } \\
\text { consumption }\end{array}$} & $<21$ units/wk & 32 & 22 & 97 & 1.78 & $0.305^{\star}$ \\
\hline & $>21$ units/wk & 1 & 3 & 3 & & \\
\hline \multirow[t]{2}{*}{ Smoking } & No & 26 & 16 & 78.8 & 1.56 & 0.25 \\
\hline & Yes & 7 & 9 & 21.2 & & \\
\hline \multirow[t]{2}{*}{ Group } & TBW & 15 & 14 & 45.5 & 0.63 & 0.60 \\
\hline & $\mathrm{PF}$ & 18 & 11 & 54.5 & & \\
\hline \multirow[t]{2}{*}{ Fracture } & Mayo 2A & 23 & 10 & 69.7 & 5.12 & 0.03 \\
\hline & Mayo 2B & 10 & 15 & 30.3 & & \\
\hline Age & $36(25.5,51)$ & $37(22.5,55)$ & & & -0.20 & $0.838 \triangle$ \\
\hline ASA grade & $1(1,1)$ & $1(1,1.5)$ & & & -0.34 & $0.738 \triangle$ \\
\hline
\end{tabular}




\begin{tabular}{|c|c|c|c|c|c|c|}
\hline & & Excellent & $\begin{array}{l}\text { Not } \\
\text { excellent }\end{array}$ & Odds(\%) & $\chi^{2} / Z / F$ & $P$ \\
\hline BMI & $22.46 \pm 3.12$ & $\begin{array}{l}22.67 \pm \\
3.03\end{array}$ & & & 0.31 & $0.58 \#$ \\
\hline
\end{tabular}

Table 8

Multivariate analysis of variables relating to treatment efficacy in 58 patients after propensity match scoring

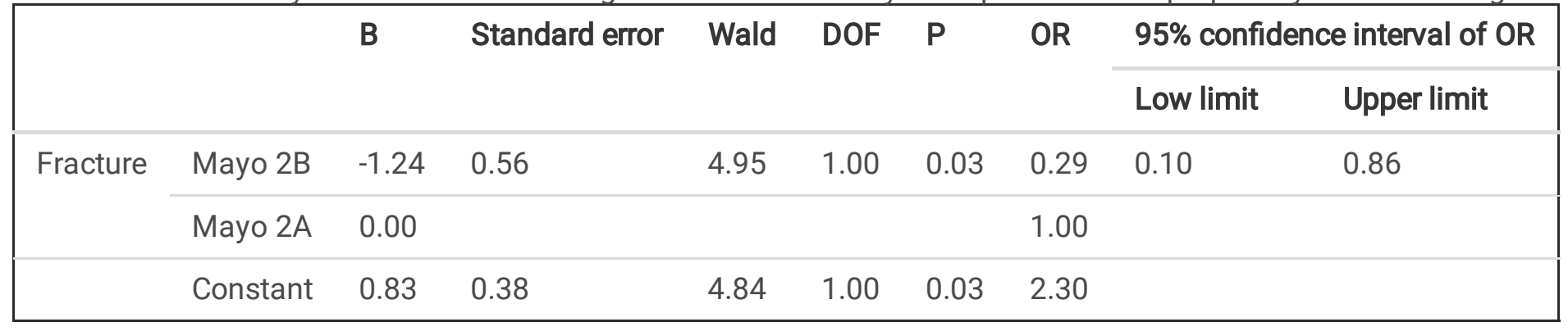

Univariate analysis and multivariate analysis of variables relating to metalwork removal in $\mathbf{5 8}$ patients after propensity score matching.

A univariate analysis of 58 patients after propensity score matching revealed that fracture type and age were correlated with metalwork removal (Table 9). Multivariate analysis demonstrated that group (treatment type) was an independent factor that affected metalwork removal of an olecranon fracture (regression coefficient $2.38>0, O R=10.77, P<0.01$ ), indicating that the risk of metalwork removal in Group TBW was 10.77 times that that in Group PF. Furthermore, multivariate analysis demonstrated that age was an independent factor that affected metalwork removal (regression coefficient $=-0.04<0, P=0.03$ ), indicating that for each additional year of age, the probability of metalwork removal is $96 \%$ of the probability of patients 1 year younger (Table 10). In other words, age is a protective factor against metalwork removal. 
Table 9

Univariate analysis of variables relating to metalwork removal in 58 patients after propensity match scoring

\begin{tabular}{|c|c|c|c|c|c|}
\hline & & \multicolumn{4}{|c|}{ Complication } \\
\hline & & No & Yes & $x^{2} / Z$ & $\mathrm{P}$ \\
\hline \multirow[t]{2}{*}{ Sex } & Men (\%) & $17(53.13 \%)$ & $15(46.87 \%)$ & \multirow[t]{2}{*}{1.24} & \multirow[t]{2}{*}{0.27} \\
\hline & Women\%) & $10(38.46 \%)$ & $16(61.54 \%)$ & & \\
\hline \multirow[t]{2}{*}{ Occupation } & Non-heavy labor & $17(42.5 \%)$ & $23(57.5 \%)$ & \multirow[t]{2}{*}{0.85} & \multirow[t]{2}{*}{0.40} \\
\hline & Heavy labor & $10(55.6 \%)$ & $8(44.4 \%)$ & & \\
\hline \multirow[t]{5}{*}{ Associated injury } & No (\%) & $9(31)$ & $20(69)$ & \multirow[t]{5}{*}{$7.2^{\star}$} & \multirow[t]{5}{*}{0.12} \\
\hline & Ipsilateral shoulder joint Injury (\%) & $6(75)$ & $2(25)$ & & \\
\hline & Ipsilateral wrist joints injury (\%) & $3(42.9)$ & $4(57.1)$ & & \\
\hline & Minor head injury (\%) & $6(66.7)$ & $3(33.3)$ & & \\
\hline & Others (\%) & $3(60)$ & $2(40)$ & & \\
\hline \multirow[t]{6}{*}{ Mechanism of injury } & No (\%) & $13(54.2)$ & $11(45.8)$ & \multirow[t]{6}{*}{$1.35^{\star}$} & \multirow[t]{6}{*}{0.90} \\
\hline & Fall from standing height (\%) & $3(37.5)$ & $5(62.5)$ & & \\
\hline & Fall from height (\%) & $6(40)$ & $9(60)$ & & \\
\hline & Motor vehicle collision (\%) & $3(42.9)$ & $4(57.1)$ & & \\
\hline & Sports (\%) & $2(50)$ & $2(50)$ & & \\
\hline & Others (\%) & & & & \\
\hline \multirow[t]{2}{*}{ Diabetes } & No (\%) & $25(44.6)$ & $31(55.4)$ & \multirow[t]{2}{*}{$2.38 *$} & \multirow[t]{2}{*}{0.21} \\
\hline & Yes (\%) & $2(100)$ & $0(0)$ & & \\
\hline \multirow[t]{2}{*}{ Dominant hand } & Left (\%) & $0(0)$ & $0(0)$ & \multirow[t]{2}{*}{ constant\# } & \\
\hline & Right (\%) & $27(46.6)$ & $31(53.4)$ & & \\
\hline \multirow[t]{2}{*}{ Side of injury } & Left (\%) & $15(41.7)$ & $21(58.3)$ & \multirow[t]{2}{*}{0.91} & \multirow[t]{2}{*}{0.42} \\
\hline & Right (\%) & $12(54.5)$ & $10(45.5)$ & & \\
\hline \multirow[t]{2}{*}{ Alcohol consumption } & $<21$ units/wk (\%) & \multirow[t]{2}{*}{$26(48.1)$} & $28(51.9)$ & \multirow[t]{2}{*}{0.80} & \multirow[t]{2}{*}{0.62} \\
\hline & $>21$ units/wk (\%) & & & & \\
\hline \multirow[t]{2}{*}{ Smoking } & No (\%) & $19(45.2)$ & $23(54.8)$ & \multirow[t]{2}{*}{0.11} & \multirow[t]{2}{*}{0.78} \\
\hline & Yes (\%) & $8(27)$ & $8(31)$ & & \\
\hline \multirow[t]{2}{*}{ Group } & TBW (\%) & $6(21)$ & $23(79)$ & \multirow[t]{2}{*}{15.59} & \multirow[t]{2}{*}{$<0.001$} \\
\hline & PF (\%) & $21(72)$ & $8(18)$ & & \\
\hline Fracture & Mayo 2A (\%) & $18(54.5)$ & $15(45.5)$ & 1.97 & 0.19 \\
\hline & Mayo 2B (\%) & $9(36)$ & $16(64)$ & & \\
\hline
\end{tabular}




\begin{tabular}{|lllll|}
\hline \multicolumn{5}{|c|}{ Complication } \\
\hline Age $(\mathrm{QSD})$ & $43(33,66)$ & $31(22,42)$ & -2.794 & 0.005 \\
\hline ASA grade (QSD) & $1(1,1)$ & $1(1,2)$ & -0.815 & 0.415 \\
\hline BMI(M \pm SD) & $23.02 \pm 0.57$ & $22.2 \pm 0.56$ & 1.02 & 0.99 \\
\hline
\end{tabular}

Table 10

Multivariate analysis of variables relating to metalwork removal in 58 patients after propensity match scoring

\begin{tabular}{|c|c|c|c|c|c|c|c|c|}
\hline & \multirow[t]{2}{*}{ B } & \multirow[t]{2}{*}{ Standard error } & \multirow[t]{2}{*}{ Wald } & \multirow[t]{2}{*}{ DOF } & \multirow[t]{2}{*}{$P$} & \multirow[t]{2}{*}{ OR } & \multicolumn{2}{|c|}{$\begin{array}{l}95 \% \text { confidence interval } \\
\text { of OR }\end{array}$} \\
\hline & & & & & & & Low limit & Upper limit \\
\hline Age & -0.04 & 0.02 & 4.86 & 1.00 & 0.03 & 0.96 & 0.93 & 1.00 \\
\hline Group TBW & 2.38 & 0.67 & 12.69 & 1.00 & $<0.01$ & 10.77 & 2.91 & 39.80 \\
\hline Group PF & 0 & & & & & 1 & & \\
\hline Constant & 0.71 & 0.83 & 0.73 & 1.00 & 0.39 & 2.04 & & \\
\hline
\end{tabular}

\section{Discussion}

Although several novel treatments appeared promising, traditional tension band wiring and plate fixation represent the most common methods for treating olecranon fractures $[1,5]$. Tension band wiring remains the gold standard within transverse simple olecranon fractures (Mayo 2A). Owing to better biomechanical compression across fractures, plate fixation was preferred for comminuted olecranon fractures[8]. However, some scholars also recommended tension band wiring for comminuted olecranon fractures (Mayo 2B) owing to the comparable cost or shorter operation time[4, 9]. There was very low-quality evidence that functional outcomes (such as the Disabilities of the Arm, Shoulder and Hand Score, the Mayo Elbow Performance Score and flexion arc) between PF and TBW were similar. This lack of difference may be the result of studies being underpowered to evaluate functional differences between TBW and PF[1]. Therefore, the treatment efficacy of the classical treatment methods for olecranon fractures, i.e., TBW and PF, is unclear, and there is no agreement as to which method provides the best outcome and should therefore be recommended. The current study aimed to examine the differences between TBW and PF using propensity score matching analysis and aimed to propose appropriate treatment methods for displaced olecranon fractures. To eliminate the influence of differences in basic factors between groups, the current study adopted the propensity scoring match method. Therefore, the comparison of the outcomes between the two groups was more reliable, which could not be achieved in most retrospective studies.

There was no difference in functional effects between Group TBW and Group PF (in terms of the MEPS, forearm arc, elbow flexion, and RTW), which was consistent with previous studies [4, 9-13]. Multivariate analysis demonstrated that the only independent factor that affected the MEPS was fracture type $(69.7 \%$ vs $30.3 \%, P=0.03)$, indicating that the severity of the olecranon fracture was negatively correlated with the treatment efficacy of olecranon fracture. As pointed out by many previous studies, the complication rate was higher in the TBW group than in the PF group[9, 14, 15], which was confirmed again in the current study in both the original 107 patients recruited to participate in this study and in the 58 patients analyzed for propensity score matching. Given the position of internal fixation on the dorsal aspect ulna, the main perceived adverse events of olecranon fractures was prominent hardware removal. It 
would be imprudent to consider symptomatic hardware trivial because it was also found to be the most common cause of hardware removal[2, 9]. Therefore, metalwork removal was adopted as the main perceived adverse events, and the potential factors affecting metalwork removal were investigated by binary logistic regression analysis. Interestingly, age and treatment options were two independent factors that affected metalwork removal from a statistical perspective. On one hand, we have reasons to believe the reliability of the higher metalwork removal rate in TBW than PF because some experts have demonstrated that plate fixation provide significantly greater compression than tension band wiring in the treatment of transverse fractures of the olecranon[8, 15], both over the whole fracture and specifically at the articular side of the fracture. Another biomechanical advantage of this method is the better holding power of plate fixation and less soft tissue stimulation of the metalwork. Although the plate fixation fare in the current study was higher than that of tension band wiring $(23519(17232,25424.5)$ vs $11943(10201,17978))$, which was similar to a previous study, the overall expense may be higher if the hospitalization and travel expenses incurred from the metalwork removal operation are considered[4, 9]. Therefore, a second operation should carry considerable weight in the initial implant decision. On the other hand, age should not be regarded as an independent factor of metalwork removal: the false positive results in the current study may be related to underpower effects and could possibly be resolved by increasing the sample size in future studies; the poor follow-up compliance of the elderly leads to a low positive record of subsequent adverse events. Conservative treatment or bone resection is still the first treatment option for low-demand elderly individuals with cancellous bone[16]. Plate fixation is still the preferred choice if internal fixation is decided for elderly individuals with olecranon fractures, owing to the more reliable biological stability in plate fixation[3, 17].

A primary disadvantage of this study was the lack of blinding of both surgeons and patients to the allocated treatment method. It is argued that this is pragmatic in routine clinical practice, as people would always be aware of their proposed treatments. A further limitation to acknowledge is the fact that multiple surgeons with different experience levels were involved over the study period and people with concomitant injuries were involved in this study, which was also pragmatic and reflective of daily clinical practice[18]. Third, we could not exclude potentially subjective decisions to remove the metalwork. However, it is not routine to remove the metalwork unless the patient is symptomatic.

We thus recommend that young patients in our department with displaced olecranon fractures (either Mayo 2A or Mayo 2B) should undergo plate fixation as the first choice when considering treatment options because the same functional score was achieved with a lower internal fixation removal rate.

\section{Conclusion}

Based on the higher metalwork removal in the tension band wiring and the same functional score compared with plate fixation, we thus concluded that plate fixation should be the first choice of treatment method for olecranon fractures to avoid potential metalwork removal, especially in younger patients with higher quality of life requirements.

\section{Abbreviations}

TBW

tension band wiring; PF:plate fixation; MEPS:Mayo Elbow Performance Score; ROM:range of elbow motion; RFR:range of forearm rotation; RTW:the time return to work; BMl:body mass index ; ASA:American Society of Anesthesiologists

\section{Declarations}

\section{Ethics approval and consent to participate}


Ethical approval was obtained from the Institutional Review Board of Guangdong Medical University, and the study conformed to the tenets of the Declaration of Helsinki.

\section{Consent to publicate}

Written informed consent for publication was obtained from all participants.

\section{Availability of data and materials}

All data generated or analysed during this study are included in this article.

\section{Competing interests}

We declare that we have no financial and personal relationships with other people or organizations that can inappropriately influence our work, there is no professional or other personal interest of any nature or kind in any product, service and/or company that could be construed as influencing the position presented in, or the review of, the manuscript entitled "More metalwork removals of olecranon fracture after tension banding wiring than plate fixation-a propensity score matching analysis".

\section{Funding}

Not applicable.

\section{Authors'contributions}

Y L『Management activities to produce metadata, scrub data, and/or maintain research data (including software code) for initial use and later re-use. Oversight and leadership responsibility for the research activity planning and execution, including mentorship. Original Draft: Preparation, creation and/or presentation of the published work, specifically writing the initial draft, including substantial translation.

LH Q:Application of statistical, mathematical, computational, or other formal techniques to analyze or synthesize study data. Management and coordination responsibility for the research activity planning and execution.

HB W:Conducting the research and investigation process, specifically performing the experiments, or data/evidence collection.

RX C:Development or design of methodology.

ZW Z:Review \& Editing: Preparation, creation and/or presentation of the published work by those from the original research group, specifically critical review, commentary, or revision, including pre- or post-publication stages.

XF W:Programming, software development; designing computer programs; implementation of the computer code and supporting algorithms; testing of existing code components.

YL H: Management and coordination responsibility for the research activity planning and execution. Assume responsibility for role assignment.

\section{Acknowledgements}

Not applicable. 


\section{References}

1. Matar HE, Ali AA, Buckley S, et al. Surgical interventions for treating fractures of the olecranon in adults. Cochrane Database of Systematic Reviews. 2014. https://doi.org/10.1002/14651858.cd010144.pub2.

2. Matar HE, Miller DJ, Duckett SP. Metalwork prominence and operative interventions for treating olecranon fractures: Systematic review of randomised controlled trials. J Long Term Eff Med Implants. 2018;28:335-45. https://doi.org/10.1615/jlongtermeffmedimplants.2019030090.

3. Duckworth AD, Clement ND, McEachan JE, et al: Prospective randomised trial of non-operative versus operative management of olecranon fractures in the elderly. Bone Joint J 99-b:964 - 72,2017. https://doi.org/10.1302/0301-620x.99b7.bjj-2016-1112.r2.

4. Powell AJ, Farhan-Alanie OM, McGraw IWW. Tension band wiring versus locking plate fixation for simple, two-part Mayo 2A olecranon fractures: a comparison of post-operative outcomes, complications, reoperations and economics. Musculoskelet Surg. 2019;103:155-60. https://doi.org/10.1007/s12306-018-0556-6.

5. Koziarz A, Woolnough T, Oitment C, et al. Surgical management for olecranon fractures in adults: A systematic review and meta-analysis. Orthopedics. 2019;42:75-82. https://doi.org/10.3928/01477447-20190221-03.

6. Di Francia R, Letissier H, Le Nen D, et al. Advantages of expulsion-proof pins in the treatment of olecranon fractures with tension band wiring: Comparison with a control group. Orthopaedics Traumatology: Surgery Research. 2019;105:1593-99. http://dx.doi.org/10.1016/j.otsr.2019.08.020.

7. Uhlmann M, Barg A, Valderrabano V, et al. Treatment of isolated fractures of the olecranon: percutaneous doublescrew fixation versus conventional tension band wiring. Der Unfallchirurg. 2014;117:614-23. https://doi.org/10.1007/s00113-013-2389-y.

8. Wilson J, Bajwa A, Kamath V, et al. Biomechanical comparison of interfragmentary compression in transverse fractures of the olecranon. J Bone Joint Surg Br. 2011;93:245-50. https://doi.org/10.1302/0301$620 x .93 b 2.24613$.

9. Duckworth AD, Clement ND, White TO, et al. Plate Versus Tension-Band Wire Fixation for Olecranon Fractures: A Prospective Randomized Trial. J Bone Joint Surg Am. 2017;99:1261-73. https://doi.org/10.2106/jbjs.16.00773.

10. Amini MH, Azar FM, Wilson BR, et al: Comparison of Outcomes and Costs of Tension-Band and Locking-Plate Osteosynthesis in Transverse Olecranon Fractures: A Matched-Cohort Study. Am J Orthop (Belle Mead NJ) 2015;44:E211-5.(the DOI of this reference could not been found in Pubmed and journal websites where the reference was published).

11. Gathen M, Jaenisch M, Peez C, et al. Plate fixation and tension band wiring after isolated olecranon fracture comparison of outcome and complications. Journal of Orthopaedics. 2020;18:69-75.

https://doi.org/10.1016/j.jor.2019.09.017.

12. Hume MC, Wiss DA. Olecranon fractures: A clinical and radiographic comparison of tension band wiring and plate fixation. Clinical Orthopaedics and Related Research 1992;229 - 35.

13. Ren YM, Qiao HY, Wei ZJ, et al. Efficacy and safety of tension band wiring versus plate fixation in olecranon fractures: a systematic review and meta-analysis. J Orthop Surg Res. 2016;11:137. https://doi.org/10.1186/s13018-016-0465-z.

14. Schliemann B, Raschke MJ, Groene P, et al: Comparison of tension band wiring and precontoured locking compression plate fixation in Mayo type IIA olecranon fractures. Acta orthopaedica Belgica 2014;80:106 - 11. (the DOI of this reference could not been found in Pubmed and journal websites where the reference was published).

15. Tarallo L, Mugnai R, Adani R, et al. Simple and comminuted displaced olecranon fractures: a clinical comparison between tension band wiring and plate fixation techniques. Arch Orthop Trauma Surg. 2014.

Page $17 / 19$ 
https://doi.org/10.1007/s00402-014-2021-9.

16. Symes M, Harris IA, Limbers J, et al. SOFIE: Surgery for Olecranon Fractures in the Elderly: a randomised controlled trial of operative versus non-operative treatment. BMC Musculoskelet Disord. 2015;16:324. https://doi.org/10.1186/s12891-015-0789-6.

17. Duckworth AD, Bugler KE, Clement ND, et al. Nonoperative management of displaced olecranon fractures in lowdemand elderly patients. Journal of Bone Joint Surgery - Series A. 2014;96:67-72. http://dx.doi.org/10.2106/JBJS.L.01137.

18. Schwartz D, Lellouch J. Explanatory and pragmatic attitudes in therapeutical trials. J Clin Epidemiol. 2009;62:499-505. https://doi.org/10.1016/j.jclinepi.2009.01.012.

\section{Figures}

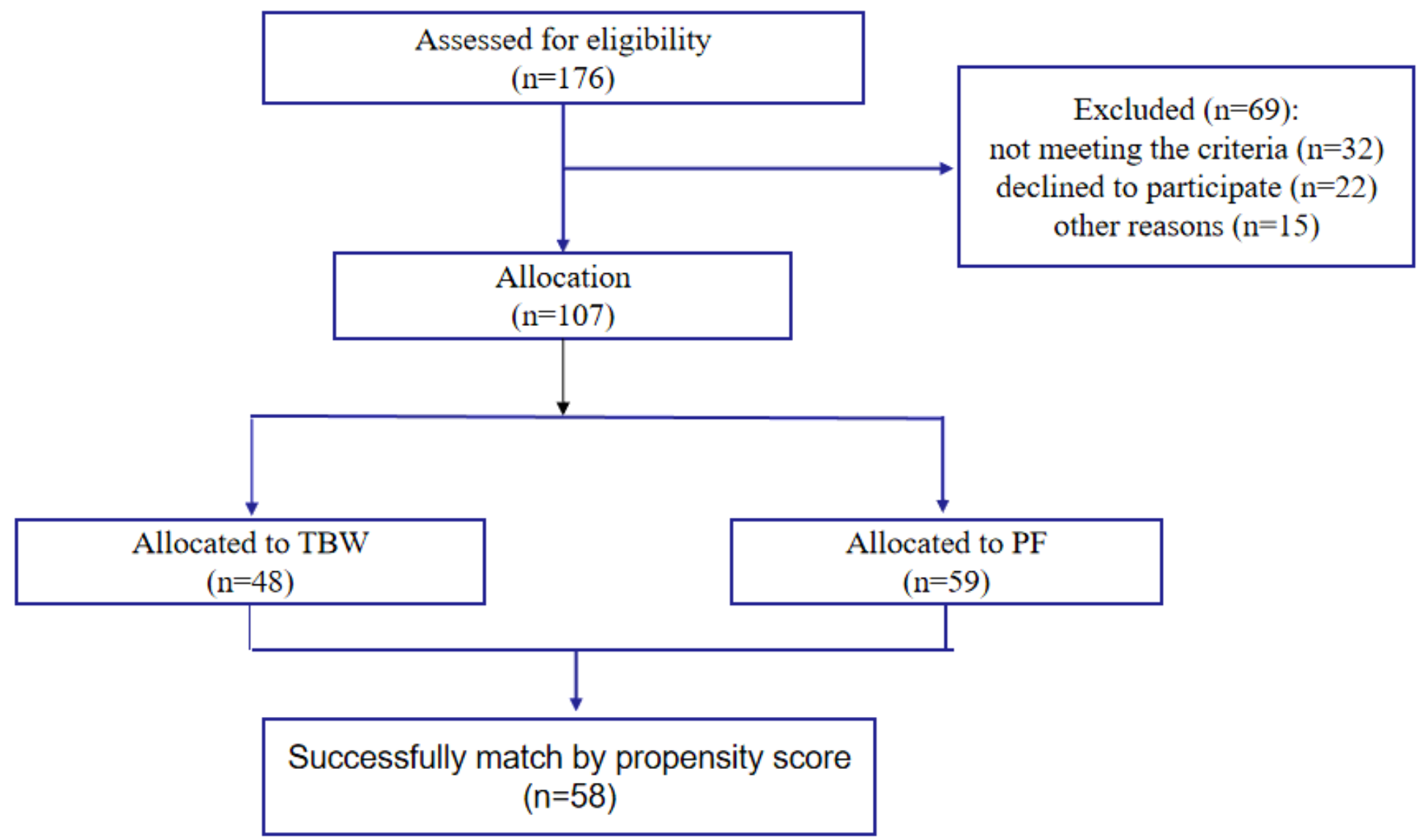

Figure 1

no legends. 


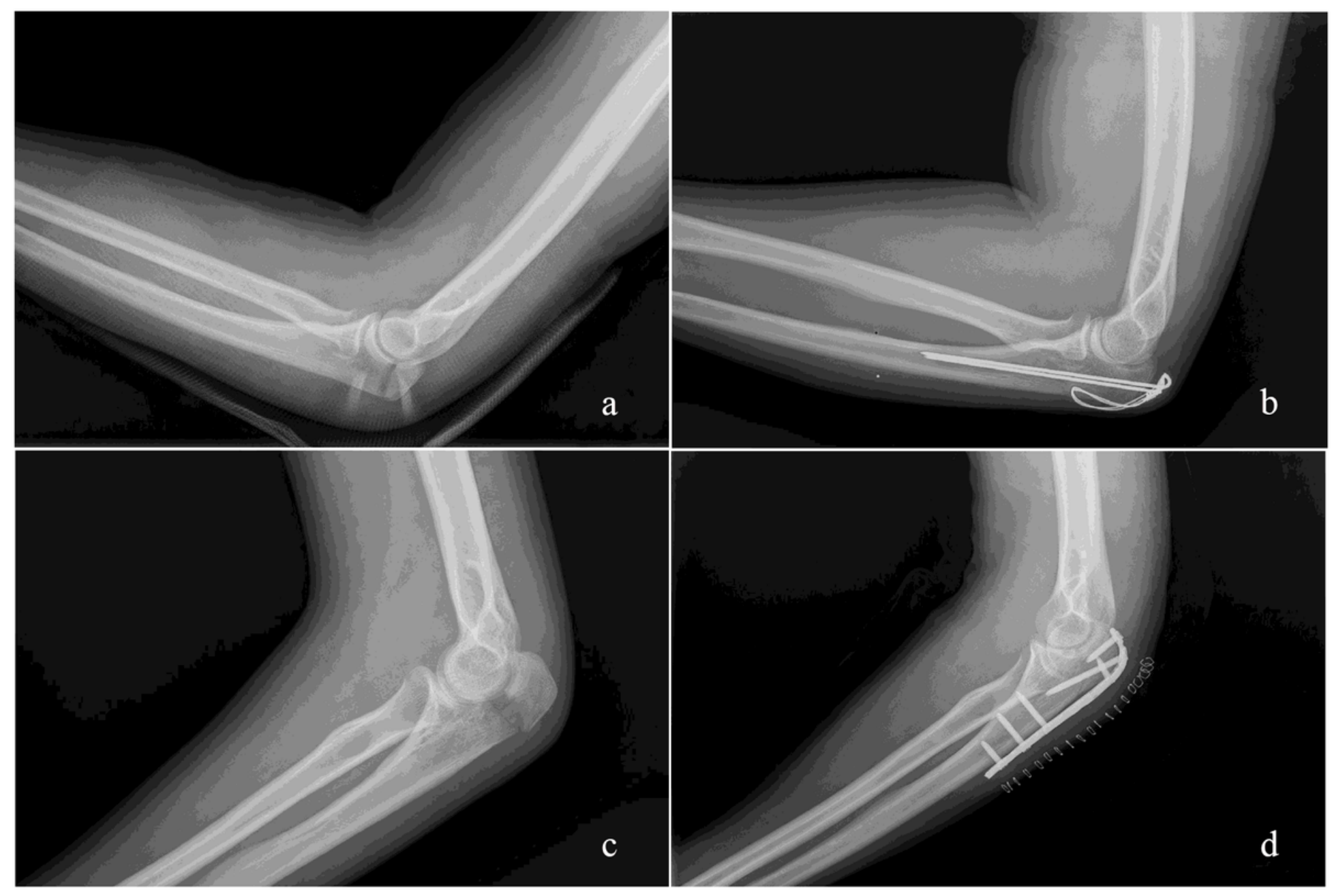

\section{Figure 2}

(A) Prepoperative lateral radiograph of elbow demonstrating Mayo 2A olecranon fracture. (B) Postoperative radiograph after tension band wiring. (C) Prepoperative lateral radiograph of elbow demonstrating another Mayo 2A olecranon fracture. (D) Postoperative radiograph after plate fixation. 